\title{
726 TUMOR TREATING FIELDS (TTFIELDS) INDUCE AN ALTERED POLARIZATION PROGRAM IN M1/M2 MACROPHAGES
}

Yiftah Barsheshet*, Boris Brant, Tali Voloshin, Alexandra Volodin, Lilach Koren, Anat KleinGoldberg, Efrat Zemer-Tov, Rom Paz, Moshe Giladi, Uri Weinberg, Yoram Palti. Novocure, Afula, Israel

Background Tumor Treating Fields (TTFields) are low intensity $(1-3 \mathrm{~V} / \mathrm{cm})$, intermediate frequency $(100-500 \mathrm{kHz})$, alternating electric fields, with demonstrated anti-mitotic effects on cancerous cells. TTFields are clinically approved for treatment of patients with glioblastoma and mesothelioma in the US and Europe. The current study aimed to examine the potential of TTFields to polarize unstimulated M0 macrophages and to regulate the phenotypes of M1 and M2 macrophages.

Methods Bone marrow-derived macrophages (BMDMs) were generated from bone marrow cells flushed from the femurs and tibias of 5-8-week-old Balb $\backslash \mathrm{C}$ mice. Unstimulated (M0 phenotype) BMDMs and BMDMs stimulated with LPS + IFN- $\gamma$ (M1 polarization) or IL-4 (M2 polarization) were treated with TTFields $(150 \mathrm{kHz})$ for 24 or 48 hours. Surface expression of the macrophage biomarker $\mathrm{F} 4 / 80$ and the activation markers CD80, major histocompatibility complex class II (MHC II), and inducible nitric oxide synthase (iNOS) were examined by flow cytometry. The heterogeneity of the stimulated macrophages was examined by a multiplexed secretion assay, capturing 13 different proteins: CXCL1 (KC), IL-18, IL-23, IL12p70, IL6, TNF- $\alpha$, IL-12p40, free active TGF- $\beta 1$, CCL22

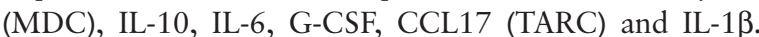

Results Application of TTFields to polarized (M1 or M2) or unpolarized BMDMs significantly increase in the percentage of CD80+/MHC IIhigh cells. M1 polarized BMDMs treated with TTFields also displayed elevation of intracellular iNOS levels. Cell supernatants of M1 and M2 stimulated BMDMs, as well as of unstimulated M0 BMDMs, displayed a pro-inflammatory secretion pattern following delivery of TTFields, with increased levels of CXCL1, IL-18, IL-23, IL-12p70, TNF- $\alpha$, IL-12p40, CCL22, G-CSF, CCL17 and IL-1 $\beta$.

Conclusions This research showed that TTFields polarized unstimulated BMDMs to the M1 phenotype, elevated the proinflammatory phenotype of M1 polarized BMDMs, and induced phenotype skewing of M2 polarized BMDMs to the M1 phenotype. These results elucidate a novel immunoregulatory role of TTFields on macrophage polarization. Future studies will aim to focus on the mechanism governing this phenotypic skewing.

http://dx.doi.org/10.1136/jitc-2021-SITC2021.726 